\title{
Antibiotic Lock Therapy
}

National Cancer Institute

\section{Source}

National Cancer Institute. Antibiotic Lock Therapy. NCI Thesaurus. Code C147519.

The use of a highly concentrated antibiotic solution for the to treatment of catheterrelated bloodstream infections. 